\title{
Research on the Organizational System of Chinese Undergraduate Universities
}

\author{
Aying Zhang ${ }^{1, a,{ }^{*},}$ Zhiping $\mathrm{Li}^{1, \mathrm{~b}}$, Zhenghong $\mathrm{Li}^{2, \mathrm{c}}$ \\ ${ }^{1}$ Harbin University, 150086 Harbin, China \\ ${ }^{2}$ Harbin Institute of Technology, 150001 Harbin, China \\ a,*zaying@sina.com, blizp2008@126.com, c273662999@qq.com
}

Keywords: Organizational system, Stability, Undergraduate, Universities.

\begin{abstract}
The coordinating elements of undergraduate universities are divided into student elements, material elements and non-material elements. Generally speaking, the internal factors of Chinese undergraduate universities are interrelated and interact with each other, which means there is a functional coupling relationship between the internal factors of Chinese undergraduate universities. This paper analyzes the functional coupling relationship between student elements and non-material elements such as cultural elements, management elements, teacher elements, and the functional coupling relationship between student elements and material elements and non-material elements. The student elements, teacher elements, management elements and cultural elements are mutually causal and mutually adjustable to become a functional coupling system. The student elements, material elements and non-material elements constitute a coupling system of mutual causation and regulation. The internal part of the Chinese undergraduate university parallelism between the elements of functional coupling, all the parallelism within the undergraduate university also has a functional coupling relationship, or the Chinese undergraduate university is a functional coupling system.
\end{abstract}

\section{Functional coupling principle}

In Jin Guantao's book "the evolution of western social structure", the principle of functional coupling in system science is put forward. Two subsystems $\mathrm{A}$ and $\mathrm{B}$, when the function of $\mathrm{A}$ is the requirement of $\mathrm{B}$ and the function of $\mathrm{B}$ is the requirement of $\mathrm{a}$, that is, when the condition of $\mathrm{A}$ is provided for the function of $\mathrm{B}$ and the condition of $\mathrm{B}$ is provided for the function of $\mathrm{A}$.
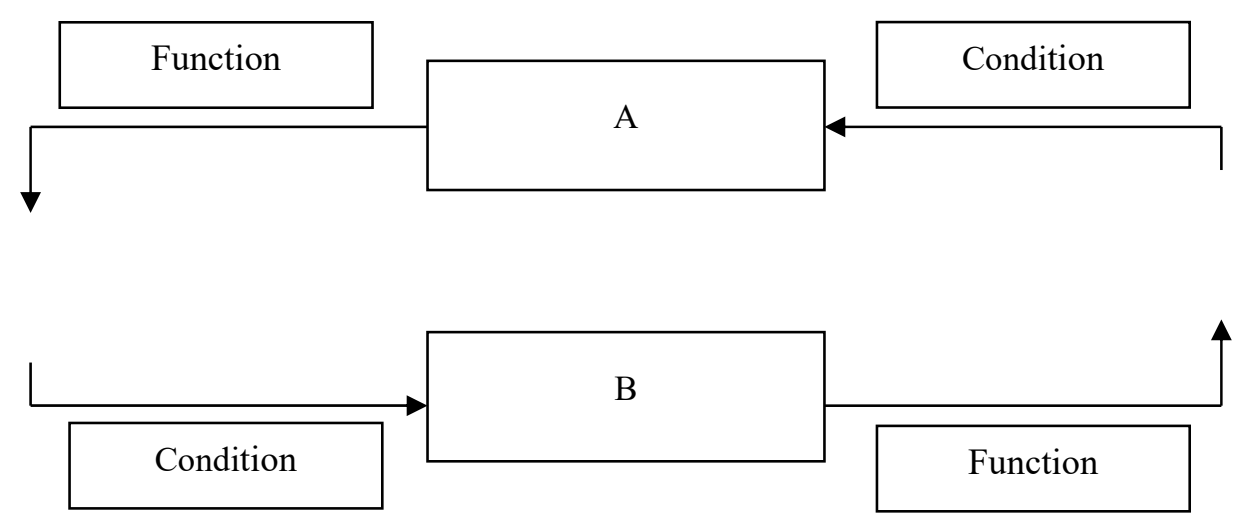

Fig. 1. The schematic diagram of functional coupling principle

As shown in Figure 1, the two subsystems form a stable system which is mutually adjustable and inseparable. Similarly, if the system has n subsystems, A1, A2 ... An, when the function of A1 is A2, 
the function of A2 is A3. When the function of A $n$ is the condition of a1, that is, when $n$ subsystems form a coupling system of mutual causation and regulation, they form a stable system.

\section{Classification of two elements in undergraduate universities}

\subsection{Juxtaposition factors of undergraduate universities}

The first function of undergraduate universities is to cultivate talents, so students are the first element; in order to cultivate students, schools should equip teachers, so teachers are the second element; according to the logic of running a school, the conditions of running a school are the third element, management system is the fourth element, and campus culture is the fifth element. These five elements are the juxtaposition elements of the development of undergraduate universities.

\subsection{The material and intangible elements of undergraduate universities}

The coordinating elements of undergraduate universities are further divided into student elements, material elements and non-material elements. In the internal juxtaposition of undergraduate universities, since students are the object of service, we consider them separately.

In the system of training students, the conditional elements (including various infrastructures, instruments, books and materials, etc.) are material.

The elements of teachers, management (principals, management teams, management policies, etc.), culture (school-running ideas, academic traditions, school atmosphere (teaching atmosphere, learning atmosphere), and so on are non-material elements.

Generally speaking, the internal factors of Chinese undergraduate universities are interrelated and interact with each other, that is, there is a functional coupling relationship between the internal factors of Chinese undergraduate universities.

This paper analyzes the functional coupling relationship between student elements and non-material elements such as cultural elements, management elements, teacher elements, and the functional coupling relationship between student elements and material elements and non-material elements.

\section{Functional coupling analysis of internal factors in undergraduate universities}

\subsection{The functional coupling between students' elements and cultural elements, management elements and teachers' elements}

According to the principle of functional coupling, the functional coupling relationship between student elements and non-material elements such as cultural elements, management elements and teachers' elements is the teacher's function, which is to carry out teaching and scientific research on the platform of subject specialty and realize the talent cultivation function of subject specialty. That is, teachers' teaching and scientific research are the conditions for students to grow up.

Students receive education and form a style of study in the process of learning. The style of study formed is one of the important components of school style (campus culture), which means that students form a style of study is the condition of school style (campus culture).

School ethos, as a universally accepted way of thinking, value judgment and behavior habits, has an important impact on the management of managers, that is, school ethos is the condition of management elements.

The academic and administrative management of managers regulate the behavior of teachers' education and teaching, that is, the management elements are the conditions of teachers' elements. 


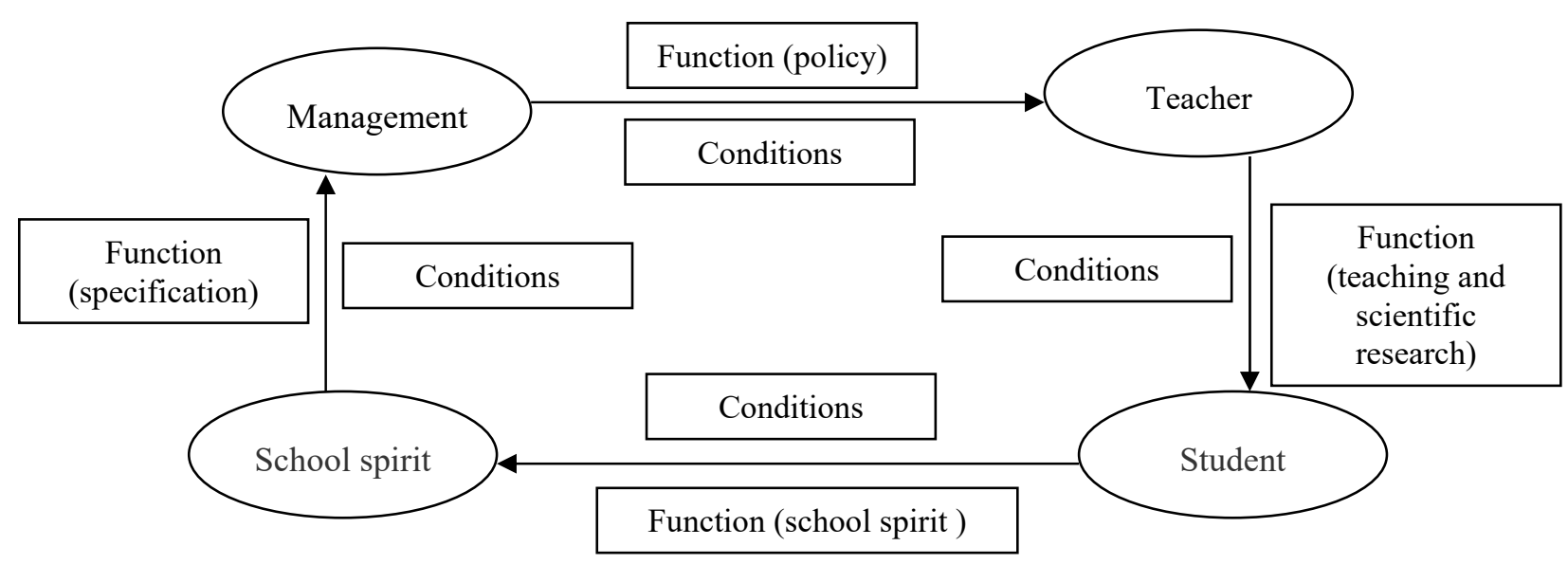

Fig. 2. The schematic diagram of functional coupling principle between internal elements of undergraduate universities

Therefore, student elements, teacher elements, management elements and cultural elements are mutually causal and mutually adjustable to become a functional coupling system.

\subsection{Functional coupling between students' essential factors and material and non-material elements in undergraduate universities}

According to the principle of functional coupling, the functional coupling relationship among student elements, material elements and non-material elements is: the condition of material elements is capital input, and the function is to provide value to meet the needs of teaching and scientific research.

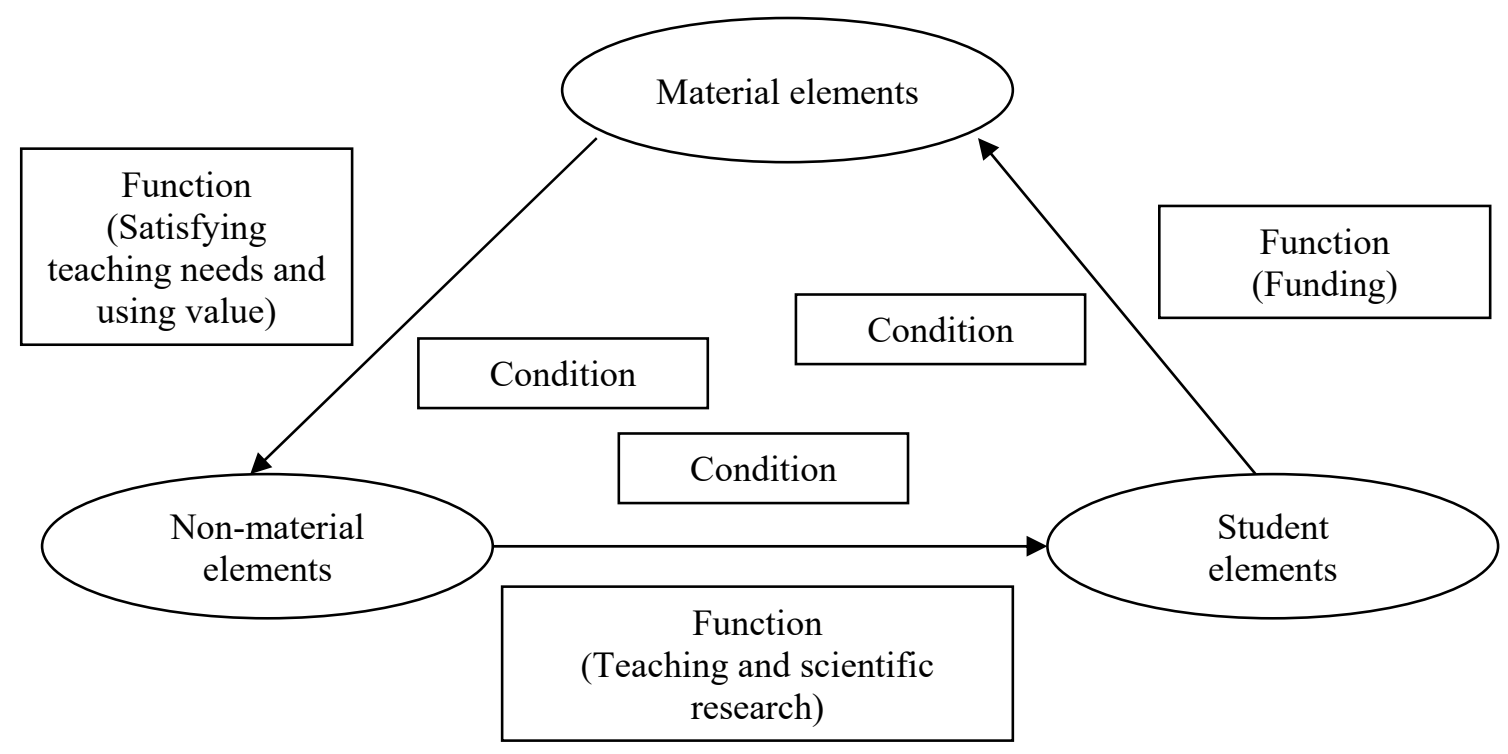

Fig. 3. The schematic diagram of functional coupling principle among student elements, material elements and non-material elements

The material elements take their functions (providing the use value to meet the needs of teaching and scientific research) as the conditions of non-material elements, which provide the conditions for 
non-material elements such as education and teaching, academic and administrative management, the cultivation of teaching and learning styles.

Non-material elements, through the teacher' education and teaching, the academic and administrative management of managers, the subtle influence of teaching style and style of study as the conditions for personnel training (student growth), implement and realize the function of personnel training.

The realization of the function of cultivating talents (students' growth) provides the school with financial support such as government input, students' payment and social donation. The funds are invested in the field of material elements, which constitute the conditions of material elements. Thus, student elements, material elements and non-material elements constitute a coupling system of mutual causation and regulation.

\section{The undergraduate university is a stable organization system}

It can be seen from the above that there is a functional coupling relationship between students' elements and non-material elements such as cultural elements, management elements and teachers' elements.

Student elements and material elements, non-material elements also constitute a functional coupling relationship, which means the internal part of the Chinese undergraduate university parallelism between the elements of functional coupling, all the parallelism within the undergraduate university also has a functional coupling relationship, or the Chinese undergraduate university is a functional coupling system.

According to the theory of systems science, all functional coupling systems are stable systems. Therefore, the undergraduate university is a stable system.

\section{Acknowledgement}

This research were financially supported by Heilongjiang Social Science Foundation (Grant No. 17SHD204).

\section{References}

[1] Z. P. Li, W. D. Wu: China Higher Education Research vol. 6 (2005), pp. 39

[2] Z. P. Li, W. D. Wu: China Higher Education Research vol. 7 (2006), pp. 56

[3] Z. P. Li, H. B. Zhang and et al: Heilongjiang Researches on Higher Education vol. 10 (2006), pp. 77

[4] Z. P. Li: Heilongjiang Researches on Higher Education vol. 3 (2005), pp. 22

[5] Z. P. Li: Education Exploration vol. 4 (2005), pp. 38

[6] Z. P. Li and et al: Journal of Harbin University vol. 10 (2005), pp. 64 